\title{
Olfactory source localization in the open field using one or both nostrils*
}

\author{
Antje Welge-Lüssen 1,\#, Gian-Luzi Looser,"\#, Birgit Westermann², \\ Thomas Hummel ${ }^{3}$ \\ ' Department of Otorhinolaryngology, University Hospital Basel, Switzerland \\ 2 Department of Neurosurgery, University Hospital Basel, Switzerland \\ ${ }^{3}$ Smell \& Taste Clinic, Department of Otorhinolaryngology, University of Dresden Medical School, Dresden, Germany
}

Rhinology 52: 41-47, 2014

DOI:10.4193/Rhino13.108

*Received for publication:

July 25,2013

Accepted: September 12, 2013

\begin{abstract}
Objective: This study aims to examine humans' abilities to localize odorants within the open field.
\end{abstract}

Methodology: Young participants were tested on a localization task using a relatively selective olfactory stimulus (2-phenylethylalcohol, PEA) and cineol, an odorant with a strong trigeminal component. Participants were blindfolded and had to localize an odorant source at $2 \mathrm{~m}$ distance (far-field condition) and a $0.4 \mathrm{~m}$ distance (near-field condition) with either two nostrils open or only one open nostril.

Results: For the odorant with trigeminal properties, the number of correct trials did not differ when one or both nostrils were used, while more PEA localization trials were correctly completed with both rather than one nostril. In the near-field condition, correct localization was possible in $72-80 \%$ of the trials, irrespective of the odorant and the number of nostrils used. Localization accuracy, measured as spatial deviation from the olfactory source, was significantly higher in the near-field compared to the farfield condition, but independent of the odorant being localized.

Conclusion: Odorant localization within the open field is difficult, but possible. In contrast to the general view, humans seem to be able to exploit the two-nostril advantage with increasing task difficulty.

Key words: odorant, olfactory, trigeminal, localization, open field, nostril

\section{Introduction}

In contrast to visual or auditory stimuli, olfactory sources are difficult to be spatially localized ${ }^{(1,2)}$, and it is still controversial whether humans are able to localize odorants at all ${ }^{(3,4)}$. This controversy is not surprising considering the different experimental setups used to examine this question. In general, egocentric and allocentric localization are differentiated. Egocentric localization is assignment of the olfactory source in relation to the main body axis whereas allocentric localization is the localization of the olfactory source within a given space. Hence in egocentric experimental setups, participants are usually fixed and motionless ${ }^{(5)}$ while in allocentric setups, participants are allowed to move at least the head, or to move within the given olfactory space $^{(5,6)}$.
Considering egocentric localization of olfactory stimuli, different experimental setups yield different results. For egocentric localization, participants rate the side of stimulation for direct, intranasally applied olfactory stimuli. Previous studies showed that in this experimental setup, relatively pure olfactory stimulants cannot be localized ${ }^{(3,7-9)}$, whereas odorants with trigeminal properties can be correctly localized with an accuracy of 82$100 \%^{(3,7,10)}$. However, when presenting the stimuli with a small time delay to each nostril or manipulating the concentration of the stimuli between both nostrils, participants were also able to correctly localize relatively selective olfactory stimulants ${ }^{(11,12)}$. Additionally, in these cases, odorants were presented at a small distance to the nostrils and participants were allowed to sniff, which is in contrast to the abovementioned experimental setups 
using intranasal stimulation. Moreover, in another experimental set-up, stimulating intranasally and examining implicit odour localization, it was hypothesized that odour localization "seems to exert an influence on human behavior" (9). All these results suggest that, as in spatial assessment of visual and auditory stimuli where time differences as short as $1 \mathrm{~ms}$ can be converted into spatial coordinates ${ }^{(13,14)}$, localization of olfactory sources is possible by exploiting a time delay or intensity differences between olfactory receptors of both nostrils. The same mechanism may support the behaviour of scent tracking in which the air sampled by the two nostrils is at least partially non-overlapping (i.e., independent samples differing in timing or intensity provide information about the location in space) ${ }^{(15,16)}$. Using two nostrils for odour sampling in scent tracking instead of only one not only increases tracking speed but also the accuracy of tracking ${ }^{(16)}$. It seems that correct olfactory localization is possible by either exploiting trigeminal properties of an odorant when applied intranasally ${ }^{(2,3,7,10,12)}$ or exploiting differences in intensity or timing, using sniffing when applied at a short distance in front of the nostrils ${ }^{(11,12,16)}$.

In humans, however, localization of olfactory sources in everyday life is normally neither egocentric nor directly comparable to the "scent-tracking" experiments performed by Szymanski and Porter et al. ${ }^{(15,16)}$. In our study, we therefore deliberately designed an experimental setup comparable to olfactory source localization in everyday life, requiring that odorants were localized within a given space. We created a modified allocentric setup to examine the effect of distance between nostrils and the olfactory source on localization accuracy (far field vs. near field). Since no suitable experimental setup exists, we performed a preliminary experiment (described below) to determine the distance between olfactory source and nostrils at which correct localization was likely to be above chance level. Using this experimental setup, we wanted to examine:

1. the accuracy of olfactory source localization in the open field in relation to the distance, far field $(2 \mathrm{~m})$ and near field $(0.4 \mathrm{~m})$, between participant and olfactory source,

2. whether the localization accuracy of pure olfactory stimulants and odorants with mixed olfactory/trigeminal properties differs,

3. whether there is a difference in the accuracy of olfactory source localization in the open space using one or two nostrils.

\section{Materials and methods}

The study was conducted in accordance with the Declaration of Helsinki; the protocol was approved by the Ethics Committee of the University of Basel. All participants provided written informed consent.

\section{Preliminary experiment}

In preliminary experiments performed with five participants, olfactory sources were arranged in a circle (in which the diameter of the circle was modified from 10, 8, 6 and finally to $4 \mathrm{~m}$ with a distance of the participant to the olfactory source at $4 \mathrm{~m}=2 \mathrm{~m}$ ) while participants were seated in the center of the circle on a rotatable chair. Participants were asked to localize the odorant placed in an odorless odorant box along the circle by sniffing while being allowed both, to move their head and to rotate their chair. At a distance of $2 \mathrm{~m}$, the number of correct localization trials was above chance when localizing a relatively pure olfactory stimulant.

\section{Participants}

Fifty-one participants [ 27 female, 24 male, mean age: 22 years, \pm 2.9 years (SD)] were included in the study. All had a negative history regarding head trauma and nasal operation, and all were non-smokers. Normosmia was ascertained in all participants using the "Sniffin'Sticks" screening test ${ }^{(17)}$. Since all subjects had negative history regarding olfactory disturbances and normosmia was a prerequisite for participation in the study nasal, endoscopy was not performed. This test battery consists of 12 odorants that have to be identified from a list of four items each using a forced-choice paradigm.

After explaining the experiments, subjects participated in two randomized localization sessions using either 2-phenylethylalcohol (PEA) or cineol as odorant. In each session, olfactory source localization in the far field was followed by the near-field experiments.

\section{Olfactory stimuli}

Two stimuli were used: PEA, a rose-like olfactory stimulus (order \# 77861; Sigma Aldrich, Buchs, Switzerland); and cineol (order\# 46090, Sigma Aldrich), a mixed olfactory/trigeminal odorant with a relatively strong trigeminal component, from here on referred to as "trigeminal odorant." In each experiment, $100 \mathrm{ml}$ of the odorant was put in a plastic dispenser called "odorant box" (size $20 \times 10 \times 4 \mathrm{~cm}$ )

\section{Far-field localization experiment}

Experiments took place in a well-ventilated room $\left(130 \mathrm{~m}^{2}\right)$ with a 3-m-high ceiling under constant conditions. A circle with a diameter of $4 \mathrm{~m}$ was arranged with small tables (height: $80 \mathrm{~cm}$ ). The tables marked 12 positions (1-12) along the circle, with each position separated by $30^{\circ}$, and served as a possible place for the odorant box (Figure 1). This setup was chosen following the preliminary experiments (as mentioned above) and is referred to from here on as "far field." Participants were blindfolded and seated on a $360^{\circ}$ swiveling chair in the center of the circle, 2 $\mathrm{m}$ from the odorant sources. For each localization trial, partici- 
pants first heard white noise via headphones. The experimenter placed the odorant box at one of the 12 positions of the circle, then opened the box and stopped the white noise to indicate the beginning of the localization trial. During subsequent searching, participants were allowed to sniff, to move their heads and to rotate their chair within $90 \mathrm{~s}$. Participants then had to indicate the direction, using a 1.8-m long pointer, where they suspected the odorant box had been placed. Participants usually started with a complete, slow, turn-around of their chair, then the chair was turned towards the suspected direction of the odorant box, and increased sniffing and head movements before making their final decision and pointing to the suspected direction. The distance from pointer to odorant box was measured and converted into degrees. The white noise was switched on again, and participants had time to relax while the odorant box was closed and carefully placed in its new position. After 3 minutes, a new localization task was started. In a random order, the odorant box was placed by the experimenter once at each of the 12 positions of the circle surrounding the participant. In half of all 12 trials, one nostril was closed using Microfoam tape (3M, St. Paul, MN, USA), which was used in an irregular order across all participants (three times right nostril closed and three times left nostril closed, respectively).

\section{Near-field localization experiment}

The 12 far-field localization trials were followed by two "nearfield" localizations (one with both nostrils, one with one nostril only). Participants went around the table circle with a distance of $40 \mathrm{~cm}$ between nose and odorant box. Otherwise, localization/indicating suspected odorant box, measurement, and angle calculation were identical (instead of using the 1.8-m pointer,

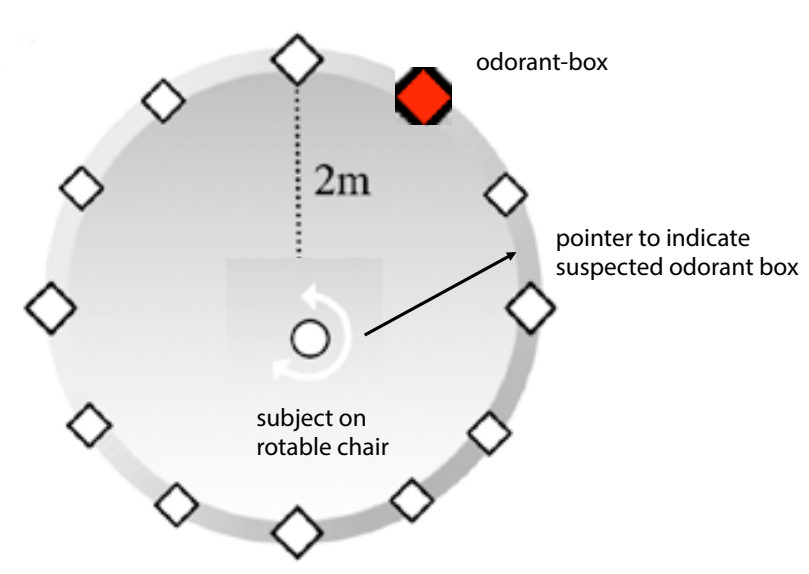

Figure 1. The experimental setup (far-field experiment). The participant sits on a rotable chair surrounded by tables on which the odorant box is placed at 12 different positions, each separated by $30^{\circ}$. The suspected localization of the box is indicated with a pointer. participants put forth their arms to indicate the odorant box). These additional near-field trials were chosen as a condition in which correct localization was assumed to be easy and very likely.

\section{Correct localization}

Correct identification was defined as localization of the odorant box within $\pm 60^{\circ}$. We chose $\pm 60^{\circ}$ to be correct due to the results of the preliminary experiments and because $\pm 90^{\circ}$ would have been the answer at chance level.

\section{Intensity rating}

Intensity of the odorant was rated by the participants after each localization trial. A rating of 0 indicated "no odorant perceived", and 10 indicated "odorant perceived as very strong". Intensity ratings were performed to ensure that the intensity did not change or drop significantly during the experiment.

This design resulted in the following four conditions: a far-field condition (distance from odour source approximately $2 \mathrm{~m}$ ) with either two nostrils open or only one nostril open, and a nearfield condition (distance from odour source approximately 0.4 $\mathrm{m}$ ) with either two nostrils open or only one nostril open. Identification was considered correct if the odorant box was localized within an angle of $\pm 60^{\circ}$.

\section{Statistical methods}

The data were analyzed using SPSS 15.0 software (SPSS Inc., Chicago, IL, USA). Analyses of variance were used (repeatedmeasures design), and $t$ tests were performed for pair-wise comparisons of conditions. Differences between conditions were considered as statistically significant at an alpha level of 0.05. Descriptive statistics are reported as means and standard error of the means (SEM).

\section{Results}

Cineol

When investigating results for cineol, this odour could be localized with one and both nostrils (one vs. both nostrils: $85^{\circ} \pm 9^{\circ}$ vs. $75^{\circ} \pm 7^{\circ}$; n.s.). During near-field localization, cineol was localized within $36^{\circ} \pm 6^{\circ}$ for both nostrils vs. $36^{\circ} \pm 5^{\circ}$ for one nostril only (n.s.). The comparison between far-field and near-field localization indicated significantly better performance during the near-field experiment ( $p<0.001$; Figure 2a).

Looking at the number of correct $\left( \pm 60^{\circ}\right)$ localization trials, during far-field localization cineol was correctly localized with both nostrils open in four or more out of six trials by 11 participants (22\%), vs. 12 participants (23.5\%) with only one nostril open (n.s.; Figure 3). In the near-field trial, cineol with one nostril closed was correctly identified by 41 participants (80\%) vs. 42 participants (82\%) with both nostrils open (n.s.). 
$2 a$

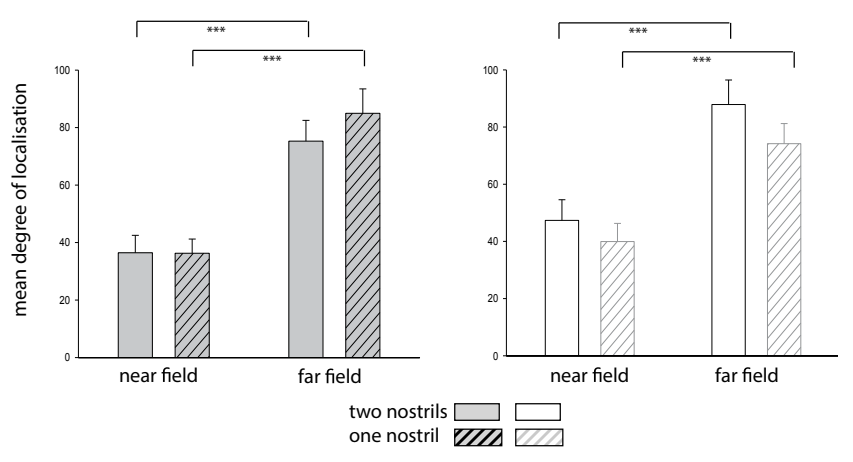

Figure 2. Cineol (a, gray bars) and PEA (b, white bars) localization with one/two nostrils in the near field vs. far field. The mean degree used to indicate the odorant box in the far field in both conditions, both nostrils (plain box) and one nostril only (coarse box), as well as the mean degree used to localize the odorant box in the near-field condition for both nostrils vs. one nostril only.

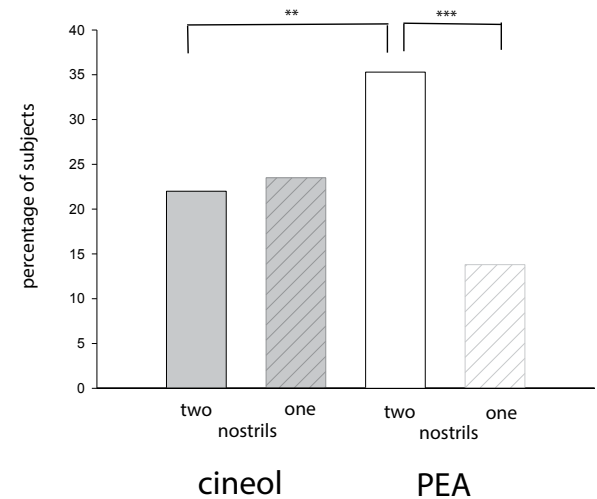

Figure 3. Percentage of participants correctly localizing the odorant box in four or more far field trials. The number of participants (presented in percentage) being able to correctly localize (within $\pm 60^{\circ}$ ) cineol (gray bars) and PEA (white bars) in four or more out of six localization trials in each condition (both nostrils vs. one nostril only).

\section{PEA}

When investigating results for PEA, the far-field trial with both nostrils open resulted in a mean localization accuracy of $88^{\circ} \pm$ $9^{\circ}$ vs. $74^{\circ} \pm 7^{\circ}$ (n.s.) in the condition with one nostril only. During the near-field trial, PEA was localized with two nostrils open with a mean accuracy of $47^{\circ} \pm 7^{\circ}$ vs. $40^{\circ} \pm 6^{\circ}$ with one nostril only (n.s.) (Figure 2b). As with cineol, only the far-field vs. near-field localization comparison revealed a significant difference $(p<$ 0.001 ), with better results obtained in the near-field condition. Looking at the number of correct $\left( \pm 60^{\circ}\right)$ localization trials, PEA was correctly localized with both nostrils open during far-field localization in four or more out of six trials by 18 participants (35.4\%) compared to 7 participants (13.8\%) with only one nostril open ( $p \leq 0.001$; Figure 3 ). In the near-field trial, PEA was correctly identified by 40 participants (78\%) with one nostril closed vs. 37 participants (72\%) with both nostrils open (n.s.).

\section{Comparison of both odorants}

During the near-field localization trial, there was no significant difference between the two odorants in both the "one nostril" (cineol: $36^{\circ} \pm 5^{\circ}$ vs. PEA: $40^{\circ} \pm 6^{\circ}$, n.s.) and "both nostrils" (cineol $36^{\circ} \pm 6^{\circ}$ vs. PEA $47^{\circ} \pm 7^{\circ}$, n.s) conditions. During far-field localization with both nostrils, cineol was identified with $85^{\circ} \pm 9^{\circ}$ vs. PEA identification of $88^{\circ} \pm 9^{\circ}$ (n.s.). In the far-field localization with one nostril only, cineol was identified with $75^{\circ} \pm 7^{\circ}$ vs. PEA $74^{\circ} \pm$ $7^{\circ}$ (n.s.).

When comparing cineol and PEA with regard to the number of correct localization trials, PEA was significantly more often correctly localized than cineol ( $p=0.002$ ) only during far-field localization with both nostrils open. With one nostril open, this difference was no longer significant (Figure 3). Among the 14 participants performing best (most correct localization trials), sex was equally distributed ( 7 male, 7 female).

\section{Intensity ratings}

The sum of the mean intensity ratings of all six sessions for both odorants with both nostrils open were compared to the sum of the six sessions with one nostril only (cineol, both nostrils vs. one: $22.5 \pm 1.4$ vs. $14.7 \pm 1.1, p<0.001$; PEA, both nostrils vs. one: $18.7 \pm 1.3$ vs. $14.6 \pm 1.0, p<0.001)$. Comparing the intensity of the odorants with one nostril closed, there was no significant difference between cineol vs. PEA (14.7 \pm 1.1 vs. $14.6 \pm 1.0$, n.s.), while in the condition both nostrils open there was a significant difference between the intensities of the two odorants (cineol vs. PEA: $22.5 \pm 1.4$ vs. $18.7 \pm 1.3, p<0.001$ ). The intensity within the session (sum of intensity during the first six trials vs. the last six trials) did not differ significantly.

\section{Discussion}

The results of the present study show that humans at a 2-mdistance are only able to localize an odorant within a given space with an accuracy of approximately $85^{\circ}$ and, as one might expect, increase this ability to an accuracy of approximately $40^{\circ}$ in the near environment (0.4-m distance). Interestingly, these results held true regardless of whether a selective olfactory stimulant (PEA) or an odorant with a predominantly trigeminal component (cineol) was used. Moreover, localization accuracy did not differ regardless whether one or two nostrils were used. Considering only the number of trials identified as correct (localization within $\pm 60^{\circ}$ ), it becomes evident again that localization in the far field is more difficult than in the near-field condition. Here, only $20-35 \%$ of the participants succeeded in correctly lo- 
calizing the odorant in the far field, compared to approximately $85 \%$ in the near-field condition.

Our findings give rise to the assumption that the localization of olfactory sources in the open space compared to the localization of direct, intranasally applied stimuli must be regarded differently. The experimental setup for these two conditions is completely different. During localization in the open space, participants are allowed to sniff, or even have to sniff, to localize the odorant. Thus, it seems that repetitive sniffing facilitates the localization process (see also ${ }^{11,12}$ ). In addition, participants were allowed to turn their heads. This altered behavioral pattern has direct consequences on the localization task, as participants are able to exploit intensity differences between both nostrils, which may in turn help to facilitate the localization of olfactory sources ${ }^{(16)}$. With respect to our results, both odorants were localized with comparable localization accuracy in both conditions [near-field: approximately $40^{\circ}$; far-field: approximately $85^{\circ}$ ], with either one or both nostrils. Although cineol was generally rated as more intense than PEA, this did not result in a significant increase in localization accuracy. It seems that both intensity and distance to the olfactory source may have contributed to the fact that neither intensity differences nor trigeminal properties could be exploited to facilitate the localization process. It is usually assumed that odours dilute and gradually diffuse away from the odorant source. However, the consideration of a decreasing gradient does not take into account that the odour dispersal process is rather chaotic, in most cases making it also very difficult to develop mathematical models to calculate the dispersion of odours ${ }^{(18,19)}$. It seems that at the distance of $2 \mathrm{~m}$ within the open space, the localization of suprathreshold odorants is possible with a similar accuracy, irrespective of their perceived intensity and property, such as being a pure olfactory or trigeminal odorant. The measured accuracy of approximately $85^{\circ}$ in the distance of $2 \mathrm{~m}$ can be considered sufficient to make a simple decision, such as to move forward or backwards, or right or left, in case of emergency. This accuracy, however, is only slightly better than the expected accuracy at chance level $\left( \pm 90^{\circ}\right)$. The distance of $2 \mathrm{~m}$, even though several individual subjects were successful in correctly localizing the odorant seems to be just at the borderline of possible correct localization.

Particularly during the far-field condition, we observed a high interindividual variance in localization accuracy. By analyzing only those trials of the far-field condition in which the participants correctly localized the olfactory source $\left( \pm 60^{\circ}\right.$, correct in four or more out of six trials), a different picture emerged. The analysis of correct trials in the far-field condition revealed that significantly more participants were able to localize the source of PEA when using two nostrils compared to trials in which one nostril was used. Since intensity of PEA was rated lower than intensity of cineol, this localization task might be considered as more difficult. It seems that in this situation, the analysis of odour samples at the two nostrils facilitated odorant source localization (20-22). It may be speculated that the comparison of bilateral odour samples is advantageous compared to the sequential sampling of odorants and comparison with memorized previous concentrations ${ }^{(23)}$. For bilateral comparison of two simultaneous samples, two separate neuronal pathways are necessary that may eventually converge. In rats, these separate pathways are only maintained through the olfactory bulb ${ }^{(24)}$, while the piriform cortex has approximately $20 \%$ cells with contralateral receptive fields in contrast to only $15 \%$ with ipsilateral receptive fields ${ }^{(25)}$. In humans, pathways remain ipsilateral from the periphery to the primary olfactory cortex, which is in contrast to the visual or auditory system ${ }^{(26)}$. There is, however, also bilateral convergence in humans in higher olfactory regions ${ }^{(27)}$. Primary olfactory regions, such as the frontal and temporal piriform cortex and the olfactory tubercle, have also been considered as a possible brain region for convergence ${ }^{(2)}$. Animal studies show that closing one nostril impairs the performance to spatially localize odorants. This supports the idea of bilateral comparison of simultaneously acquired samples as a localization strategy ${ }^{(28)}$. In this respect, our results are in line with these data and the results of Porter et al. ${ }^{(16)}$ demonstrating that scent tracking of a relatively pure olfactory stimulant is faster and more accurate when two nostrils are used compared to one nostril. In contrast to the study of Porter et al., we not only used an olfactory stimulant, PEA, but additionally tested source localization of a bimodal trigeminal/ olfactory stimulant, cineol. Interestingly, looking at the number of correct localizing trials of cineol sources, no differences between the use of one or two nostrils were found. Cineol was perceived as more intense than PEA. One might speculate that the advantage of two nostrils over one appears with increasing difficulty of the localization task. Although PEA was perceived as less intense, this would explain why PEA was correctly localized more often with two nostrils than with one. Another explanation could be that some of the participants are especially sensitive to odours, comparable to "super-tasters" (29).

\section{Study limitations}

The experimental setup in our study is new and was selected because it appeared to resemble daily life situations. Especially for this setup odorant dispersion and diffusion has to be taken into account. Regarding the chaotic odorant distribution and the fact that odorant concentration within odorant plumes is not equally distributed ${ }^{(21,30)}$, spatial concentration gradients are very difficult to obtain. Whether intensity ratings mirror these concentration gradients within odorants plumes remains speculative. The exact dispersion patterns of PEA and cineol in our setup have not been determined and were assumed to be similar. Even though experimental conditions were kept constant, possible gradient differences and differences in dispersion patterns could 
also explain why the number of correct trials for PEA was higher than for cineol using both nostrils, irrespective of the perceived intensity. Even though this was not the focus of our study, this topic may need further investigation.

Although our data seem rather robust, some confounds possibly influenced our results. We did not include any trials using an odorless fluid like water since we did not expect answers to be different from chance level; Intensity measurements revealed that cineol was perceived as slightly more intense than PEA, although this did not facilitate localization accuracy. Due to our preliminary experiments, we chose a localization angle of $\pm 60^{\circ}$ as correct, thus reducing the number of participants who were able to correctly localize odorants to $20 \%$. Although no other comparable experimental setups exists, these data reveal that the majority of subjects need to be closer than $2 \mathrm{~m}$ to an odorant source to identify the localization correctly within $\pm 60^{\circ}$ or better.

\section{Conclusion}

The results of our study show that both olfactory and trigeminal stimulants can be localized at a distance of $2 \mathrm{~m}$ in the open space with an accuracy within $85^{\circ}$, irrespective of using one or two nostrils. The localization accuracy becomes significantly bet- ter at shorter distances. However, considering an accuracy of \pm $60^{\circ}$ as correct, the number of participants able to do so drops to $20 \%$ for the distance of $2 \mathrm{~m}$. With increasing difficulty, i.e., lower concentration of the olfactory stimulus, two nostrils appear to provide an advantage over one nostril. There is a small number of participants, however, that are well capable of localizing odorants, and it remains speculative at this moment whether these participants (possibly "super smellers") would outperform other participants in terms of general olfactory sensitivity or in general spatial abilities.

\section{Acknowledgement}

This work was support by a grant of Deutsche Forschungsgemeinschaft (DFG) to TH (SPP 1392: HU 441/10-1)

\section{Authorship contribution}

AWL: Conception and study design, statistical analysis, revising manuscript; GLL : acquisition of data, drafting of article; BW : critically revising the article; $\mathrm{TH}$ : Conception and study design, statistical analysis, reviewed final version of manuscript

\section{Conflict of interest}

None to declare.

\section{References}

1. Schifferstein HNJ, Smeets MAM, Postma A Comparing location memory for 4 sensory modalites. Chem Senses. 2010; 35: 135-145.

2. Porter J, Anand T, Johnson B, Khan RM, Sobel N. Brain mechanisms for extracting spatial information from smell. Neuron. 2005; 47: 581-592.

3. Kobal G, Van Toller S, Hummel T. Is there directional smelling? Experientia. 1989; 45: 130-132.

4. Negoias S, Aszmann O, Croy I, Hummel T. Localization of odors can be learned. Chem Senses. 2013; 38: 553-562.

5. Stoddart DM. External nares and olfactory perception. Experientia. 1979; 35: 1456 1457

6. Wallace DG, Gorny B, Whishaw IQ. Rats can track odors, other rats, and themselves: implications for the study of spatial behavior. Behav Brain Res. 2002; 131: 185-192.

7. Frasnelli J, Charbonneau G, Collignon O Lepore F. Odor localization and sniffing. Chem Senses. 2009; 34: 139-144.

8. Radil T, Wysocki CJ. Spatiotemporal masking in pure olfaction. Ann N Y Acad Sciences. 1998; 855: 641-644.

9. Moessnang C, Finkelmeyer A, Vossen A Schneider F, Habel U. Assessing implicit odor localization in humans using a cross-modal spatial cuing paradigm. PLoS ONE. 2011;6:e29614, doi:10.1371/journal. pone.0029614.
10. Kleemann AM, Albrecht J, Schöpf V, et al Trigeminal perceptioin is necessary to localize odors. Physiol Behav. 2009; 97:: 401-405.

11. von Békésy G. Olfactory analogue to directional hearing. J Appl Physiol. 1964; 19: 369373.

12. Schneider RA, Schmidt CE. Dependency of olfactory localization on non-olfactory cues. Physiol Behav. 1967; 2: 305-309.

13. Lord Rayleigh. On our perception of sound direction. Philos Mag. 1907; 13: 214-232.

14. McAlpine D. Creating a sense of auditory space. J Physiol. 2005; 566: 21-28.

15. Szymanski JS. Zur Lokalisation der osmatischen Reize. Pflügers Arch. 1920; 181: 310315.

16. Porter J, Craven B, Khan RM, et al. Mechanisms of scent-tracking in humans. Nat Neurosci. 2007; 10: 27-29.

17. Hummel T, Konnerth CG, Rosenheim K Kobal G. Screening of olfactory function with a four-minute odor identification test reliability, normative data, and investigations in patients with olfactory loss. Ann Otol Rhinol Laryngol. 2001; 110: 976-981.

18. Gardiner JM, Atema J. The function of bilateral odor arrival time differences in olafctory orientation of sharks. Curr Biol. 2010; 20: 1187-1191.

19. De Melo Lisboa H, Guillot J-M, Fanlo J-L, Le Cloirec P. Dispersion of odorous gases in the atmosphere - part I: modeling approaches to the phenomenon. Sci Tot Environ. 2006;
361: 220-228.

20. Johnsen PB, Teeter JH. Spatial gradient detection of chemical cues by catfish. J Comp Physiol. 1980; 140: 95-99.

21. Atema J. Eddy chemotaxis and odor landscapes: Exploration of nature with animal sensors. Biol Bull. 1996;191:129-138.

22. Wilson DA, Sullivan RM. Respiratory airflow pattern at the rat's snout and an hypothesis regarding its role in olfaction. Physiol Behav. 1999; 66: 41-44.

23. MacNab RM, Koshland DEJ. The gradient sensing mechanism in bacterial chemotaxis. Proc Natl Acad Sci USA. 1972; 69: 25092512

24. Wilson DA. Binaral interactions in the rat piriform cortex. J Neurophysiol. 1997; 78: 160-169.

25. Wilson DA. Receptive fields in the rat piriform cortex. Chem Senses. 2001; 26: 577 484

26. Gottfried JA. Smell: central nervous processing. In: Hummel T, Welge-Lüssen A, eds. Taste and Smell. An Update.Basel: Karger; 2006. 44-69.

27. Savic I, Gulyas B. PET shows that odors are processed both ipsilateral and contralaterally to the simulated nostril. NeuroReport. 2000; 11: 2861-2866.

28. Rajan R, Clement JP, Bhalla US. Rats smell in stereo. Science. 2006; 311: 666-670.

29. Prescott J, Ripandelli N, Wakeling I. Binary tast mixtures interactions in prop non- 
tasters, medium-tasters and super-tasters. Chem Senses. 2001; 26: 993-1003.

30. Webster DR. Structure of turbulent chemical plumes. In: Wodfin RL, ed. Trace chemical sensing of explosives.New York: John Wiley and Sons; 2007. 109-129.
Prof. Dr. Antje Welge-Lüssen

University Hospital Basel

Department of Otorhinolaryngo-

logy

Petersgraben 4

$\mathrm{CH} 4031$ Basel

Switzerland
Tel: +41-61-265 4109

Fax: +41-61-265 4029

E-mail: antje.welge-luessen@usb.ch 